\title{
Microstructure anisotropy in polyolefin flexible foams
}

\author{
M Antunes, D Arencón, V Realinho and J I Velasco' ${ }^{1}$ \\ Centre Català del Plàstic. Departament de Ciència dels Materials i Enginyeria \\ Metal.lúrgica. Universitat Politècnica de Catalunya. C/Colom 114. E-08222 Terrassa, \\ Barcelona, Spain. \\ ${ }^{1}$ E-mail: jose.ignacio.velasco@upc.edu
}

\begin{abstract}
The use of polyolefin flexible foams with typical thicknesses between 1 and $3 \mathrm{~mm}$ produced by a physical foaming extrusion process is nowadays quite widespread in the packaging sector. Their high flexibility and closed-cell structure allows them to show good energy absorption properties under low loading conditions. Although the compressive response of these materials is well known, the inner microstructure developed during processing induce a high anisotropy that is responsible for their direction-dependent tensile and fracture behaviours. In this work, two different polyolefin-based foams, with densities ranging from 20 to $45 \mathrm{~kg} / \mathrm{m}^{3}$, were studied. The induced microstructure anisotropy was characterized by microRaman. With this technique, the relative orientations of both crystalline and amorphous phases in the foam's base polymer could be determined and thus related to their mechanical properties measured in the different directions.
\end{abstract}

\section{Introduction}

Polyolefin-based flexible foams, in the form of sheets with typical thicknesses ranging from 1 to $3 \mathrm{~mm}$ obtained by a physical foaming direct extrusion process, are commonly employed in packaging applications. Due to their closed-cell type of cellular structure, alongside polyolefin's high flexibility, they show improved impact energy absorption properties, enabling their use for the protection of household products.

Several studies have been reported on the structure-property relationship of closed-cell foams [1-3]; among the most important factors are the foam density, chemical composition, type of gas enclosed in the cells and polymer morphology. Processing and foaming may strongly affect the polymer's morphology, ultimately leading to preferential crystal orientations.

Traditionally, diffraction techniques such as WAXD and SAXD have been employed to study the orientation level of polymers [4-6]. Nevertheless, the main drawback is that these techniques use Xray beam sizes up to several millimeters, thus limiting the possibility of evaluating the response of single elements present in the material (such as crystal orientations in the cell faces or edges in the case of foams), the obtained information only giving an average value for the whole foam.

An alternative way of studying the crystallinity and orientation of polymeric foams is provided by Raman spectroscopy [7-8], as some vibrational modes can be related to morphologic structural differences of the base polymer. Depending on the laser beam size, the measurements may be carried 
out in surface areas as small as $1 \mu \mathrm{m}^{2}$, commonly known as micro-Raman, providing consistent and reliable information about both structural and morphological local changes.

The main aim of this work is to use Raman spectroscopy to determine the crystallinity and orientation level of two polyolefin-based foams, relating the results to the tensile mechanical behaviour.

\section{Materials}

Two commercial foamed sheets, kindly provided by OK Company (Spain), based on polyethylene and polypropylene and respectively with densities of 20 and $45 \mathrm{~kg} / \mathrm{m}^{3}$, were studied. Both samples had a nominal thickness of $2 \mathrm{~mm}$. These foamed sheets were produced by a direct extrusion physical foaming process using a tandem extruder, the physical blowing agent being added in the first extruder to the molten polymer, polymer and gas being progressively mixed and cooled in the second extruder to produce the foamed sheets by sudden decompression at the exit of the extrusion die.

\section{Experimental procedure}

\subsection{Differential Scanning Calorimetry (DSC)}

DSC measurements were performed using a Perkin Elmer Pyris 1 calorimeter with samples typically weighting around $4.0 \mathrm{mg}$. Once erasing the sample's thermal history $\left(4 \mathrm{~min}\right.$ at $\left.200{ }^{\circ} \mathrm{C}\right)$, cooling $(200$ to $40{ }^{\circ} \mathrm{C}$ ) and subsequent heating $\left(40\right.$ to $200{ }^{\circ} \mathrm{C}$ ) cycles were conducted at $10^{\circ} \mathrm{C} / \mathrm{min}$. All runs were carried out applying a constant rate of dried nitrogen.

\subsection{Fourier-Transformed Infrared Spectroscopy (FT-IR)}

Infrared spectra were obtained using a Nicolet Thermo Scientific 6700 spectrometer in FT-IR/ATR, i.e., Infrared Spectroscopy in the Attenuated Total Reflection Mode (Smart Orbit). Spectra were collected in continuous scan mode with a $4 \mathrm{~cm}^{-1}$ spectral resolution in the $4000-400 \mathrm{~cm}^{-1}$ range using 32 scans.

\subsection{Raman Spectroscopy}

Raman spectra were recorded in a Jobin Yvon T64000 spectrometer using a He/Ne Coherent Innova 300 laser $($ Temperature $=140 \mathrm{~K}$ ) operating at a wavelength of $633 \mathrm{~nm}$ and a power of $2.5 \mathrm{~mW}$ at the sample. All measurements were made in a backscattering geometry, using both a $\times 10$ (Raman mode) and $\times 50$ microscope objectives (micro-Raman), the last one with a spatial resolution of $1 \mu \mathrm{m}^{2}$.

Single point spectra were recorded for all the foams using a $0.4 \mathrm{~cm}^{-1}$ spatial resolution and $300 \mathrm{~s}$ of accumulation time. In order to assess the most characteristic Raman bands for both the PE and PP foams, the spectra of the first one were collected from 1000 to $1550 \mathrm{~cm}^{-1}$ and in the case of PP from 600 to $1600 \mathrm{~cm}^{-1}$.

Micro-Raman experiments were performed pointing the beam perpendicularly to the edge's centre and cell face in each of the considered planes: (MD,TD), (MD,ED) and (TD,ED), as seen in the schematic shown in Figure 1. Three measurements were done for each experimental set-up, the average value being used for characterization and comparison purposes.

The several PE and PP foamed samples Raman spectra were processed subtracting the respective linear baseline and using the convolution of Lorentzian and Gaussian curves in order to evaluate the position, intensity and area of each of the most characteristic bands. Both PE and PP's crystalline and amorphous contents were determined using the intensity of the respective characteristic peaks determined from the previously mentioned method. 


\subsection{Tensile tests}

Tensile tests were carried out at room temperature using dumbbell specimens (type IV, ASTM-D638) directly cut out from the foamed sheets considering the two main foaming directions (MD and TD), as shown in Figure 1, at a crosshead speed of $2 \mathrm{~mm} / \mathrm{min}$ on a universal testing machine (Galdabini Sun 2500).

\subsection{Scanning Electron Microscopy}

The cellular structure of the foams was assessed using a JEOL JSM-5610 scanning electron microscope (SEM). Samples were fractured at low temperature and made conductive by sputtering deposition of a thin layer of gold.

(a)

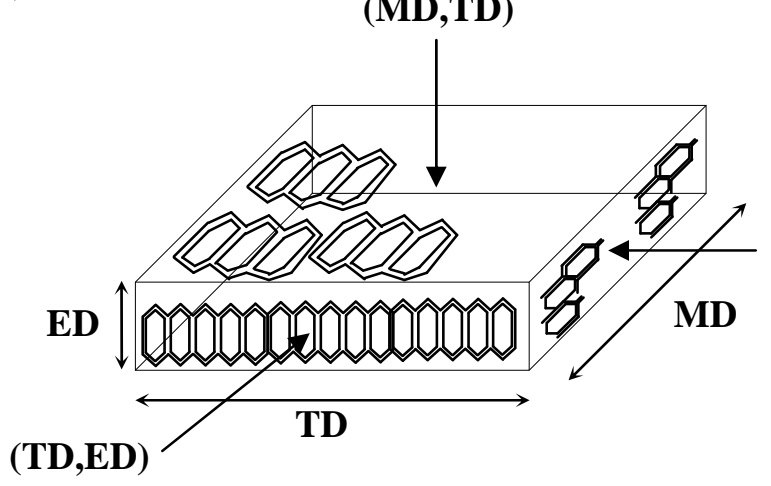

(c)

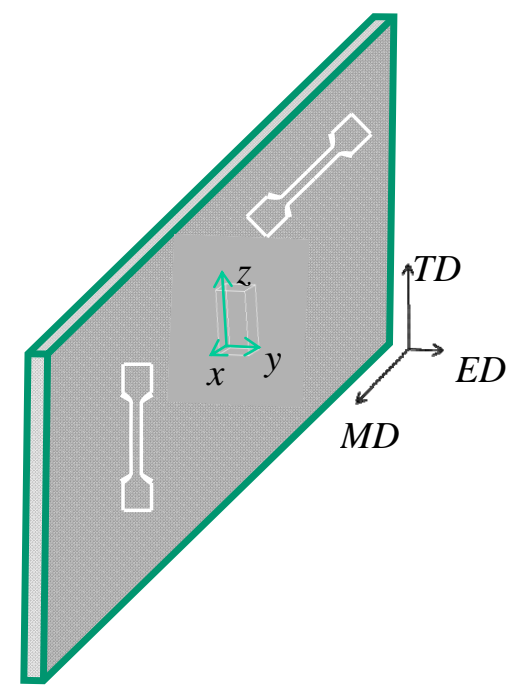

(b)

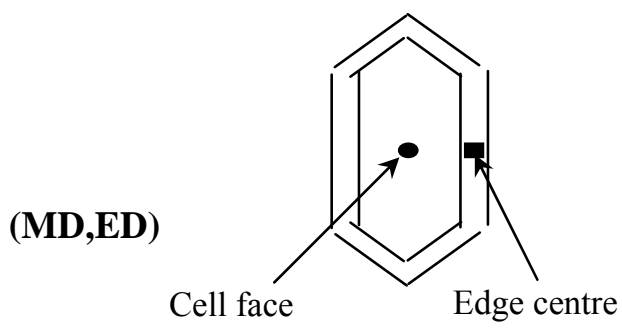

(d)

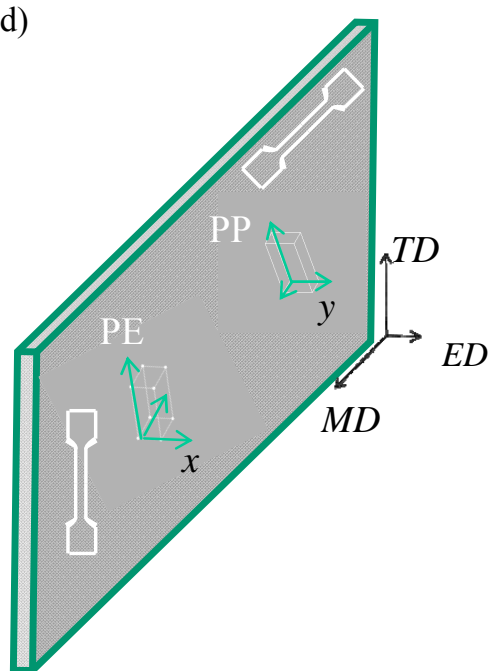

MD: Machine Direction

TD: Width direction

ED: Thickness Direction

Figure 1. Schematic showing Raman beam pointing (a) plane directions and (b) cell zones and dumbbell tensile specimen directions for (c) PE and (d) PP-based foams. 


\section{Results and discussion}

\subsection{Chemical composition and cellular structure}

4.1.1. DSC analysis. Figure 2 shows the recorded thermograms for the PE and PP foamed samples. The characteristic melting peaks of both polyethylene $\left(T_{\mathrm{f}, \mathrm{I}}=108^{\circ} \mathrm{C}\right)$ and polypropylene $\left(T_{\mathrm{f}, \mathrm{II}}=158^{\circ} \mathrm{C}\right)$ can be observed. The low value of PP's melting point allows us to say that it is a random copolymer. Moreover, small amounts of EVA, in the case of the PE foam, and a polyethylene-co-1-octene, in the case of the PP one, have also been revealed, the first of which assessed from the crystallization peak observed at $69^{\circ} \mathrm{C}$ and the second one at $86^{\circ} \mathrm{C}$. Crystallinity values obtained from the melting signals are similar for both materials: $34.2 \%$ for PE and $35.4 \%$ for PP.
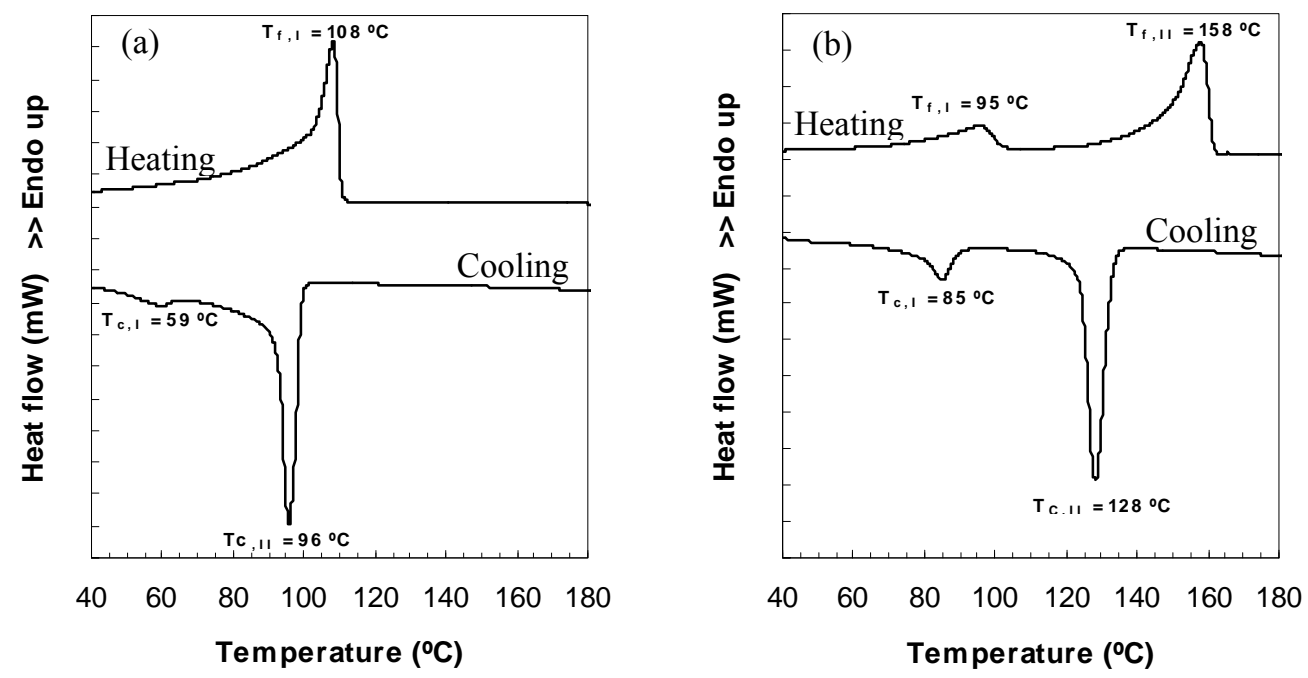

Figure 2. DSC curves for the (a) PE and (b) PP foams.

4.1.2. FT-IR analysis. The polyolefinic nature of both foams results in similar FT-IR spectra, as shown in Figure 3. Nevertheless, some differences are found in the $800-1450 \mathrm{~cm}^{-1}$ region, attributed to the presence of EVA $(\mathrm{C}=\mathrm{O}$ group) and polyethylene-co-1-octene respectively in the $\mathrm{PE}$ and $\mathrm{PP}$ based foams.

First of all, if we analyze PE's FT-IR it is possible to notice the presence of the characteristic 2950 and $1460 \mathrm{~cm}^{-1}$ strong bands and $725 \mathrm{~cm}^{-1}$ medium intensity band, all related to the $\mathrm{C}-\mathrm{H}$ stretching, deformation and rocking vibrations [9]. Additional weaker bands are found near 1380 and $1365 \mathrm{~cm}^{-1}$, related to main backbone branching and usually observed in low density polyethylene (LDPE). Although somewhat hindered due to the fact that it is added in a small amount to the LDPE-based matrix, the typical $\mathrm{C}=\mathrm{O}$ stretching vibration of the acetate group $\left(-\mathrm{O}-\mathrm{CO}-\mathrm{CH}_{3}\right)$ observed at around $1660 \mathrm{~cm}^{-1}$ allows us to say, alongside DSC results, that the PE foam has a small amount of an ethylene vinyl acetate copolymer (EVA), commonly used to increase the flexibility of the foamed material.

Alongside the strong bands observed at around 2950, 1460 and $1380 \mathrm{~cm}^{-1}$ and some sharp bands of medium intensity $\left(1155\right.$ and $\left.970 \mathrm{~cm}^{-1}\right)$, all typical of isotactic PP, we can also see some of PE's characteristic peaks, mainly the one at $725 \mathrm{~cm}^{-1}$, indicating us an ethylene-based phase to be also present in the material, as expected considering DSC results. 


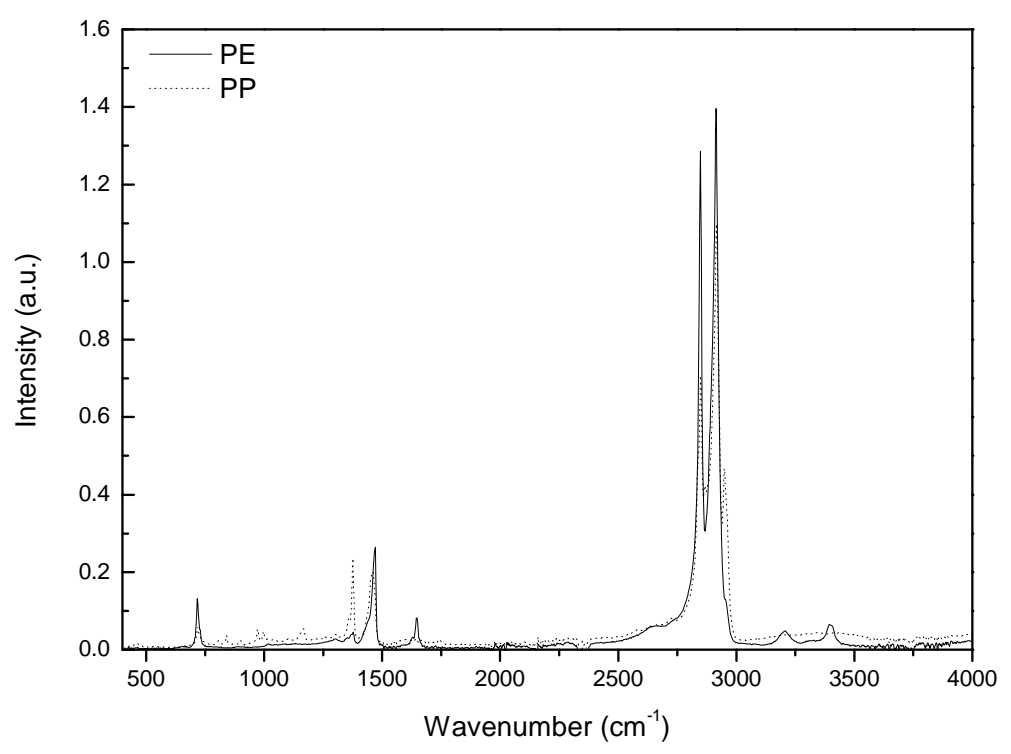

Figure 3. FT-IR spectra of the PE and PP based foams.

4.1.3. Cellular structure characterization. Fracture surfaces obtained under brittle conditions were observed using SEM (Figure 4), with differences being found regarding cell shape between the two studied foams, the PE foam presenting a more isometric-like cell shape (Figure 4(a)), with aspect ratios (AR), i.e., the quotient between the highest and smallest characteristic cell sizes, slightly higher than 1, contrarily to the clearly elongated cells of the PP one (Figure 4(c)).
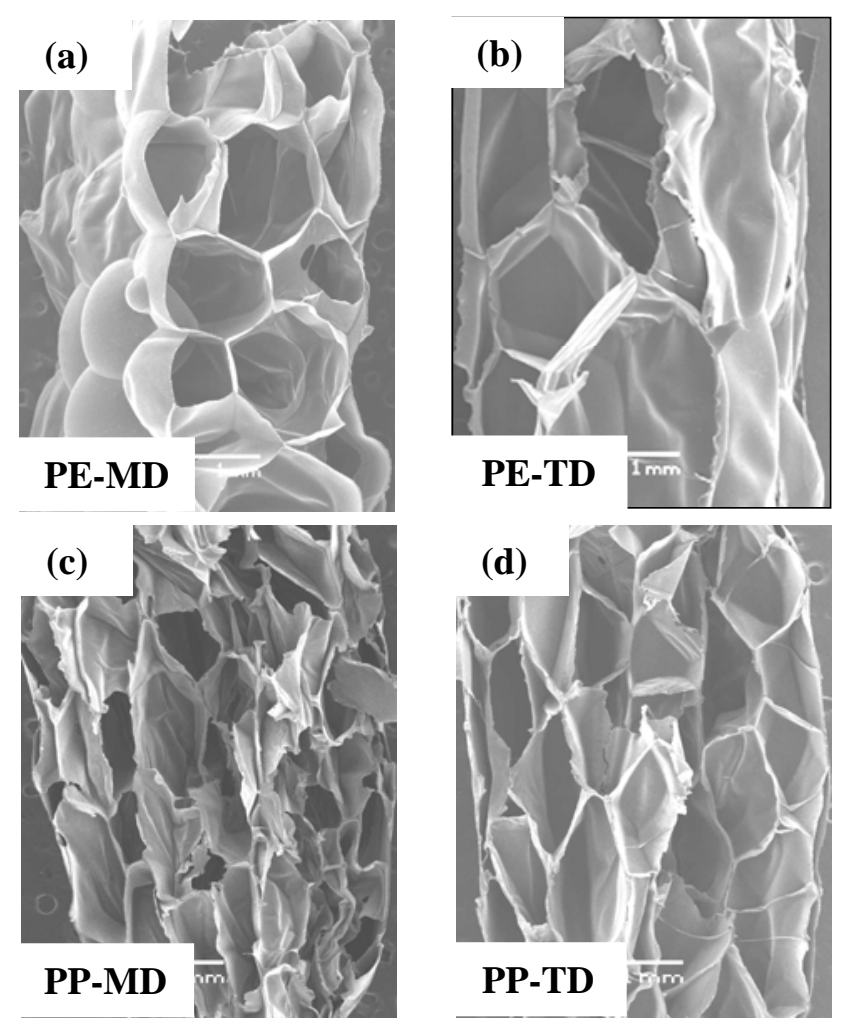

Figure 4. SEM micrographs showing the cellular structure of PE in (a) MD and (b) TD and of PP in (c) MD and (d) TD directions. 
The presence of a second solid phase was found for both foams as seen from the highmagnification SEM micrographs shown in Figure 5, once again confirming the results obtained by DSC and FT-IR.
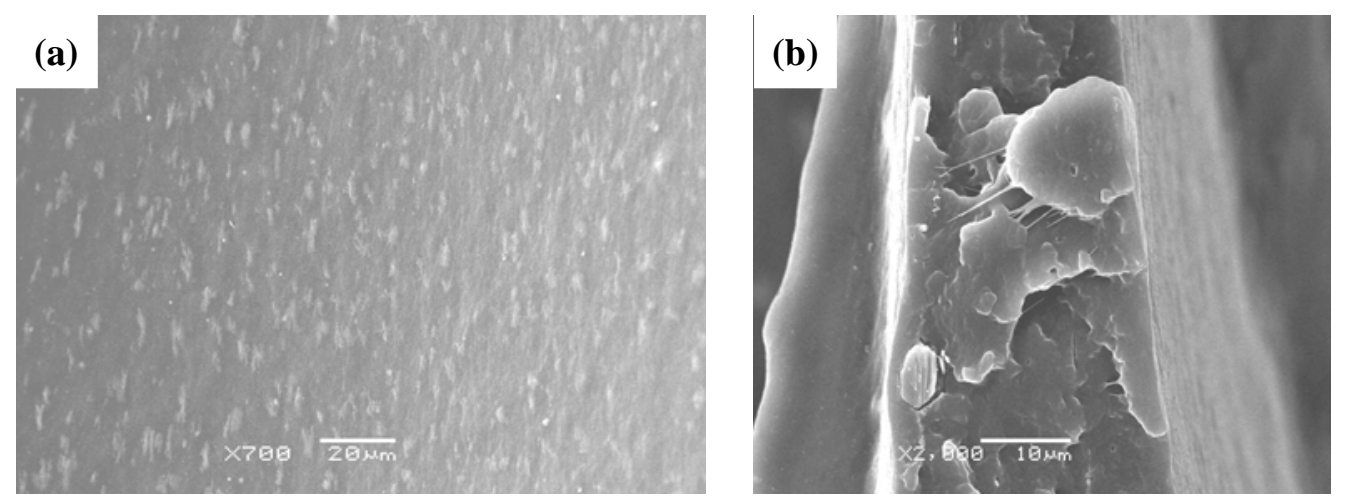

Figure 5. SEM micrographs showing the presence of an ethylene-based phase in the PP foams.

\subsection{Molecular and crystalline orientations}

4.2.1. Amorphous phase orientation by Raman spectroscopy. It is reported in the literature that the fraction of amorphous $\left(I_{\mathrm{aPE}}\right)$ and crystalline $\left(I_{\mathrm{cPE}}\right)$ phases can be calculated for PE considering the intensity of the Raman characteristic fundamental vibrational bands located at 1270, 1295, 1305 and $1416 \mathrm{~cm}^{-1}$, the last of which thought to be a direct measurement of polyethylene's orthorhombic crystallinity [10]:

$$
I_{c P E}=\frac{I_{1416}}{\left(I_{1295}+I_{1305}+I_{1270}\right) \times 0.45} \times 100,
$$

where 0.45 , related to the presence of an intermediate phase in the crystalline boundary, was found through experiments [11].

Similarly, the amorphous fraction $\left(I_{\mathrm{aPE}}\right)$ can be calculated considering the characteristic band at $1305 \mathrm{~cm}^{-1}$ :

$$
I_{a P E}=\frac{I_{1305}}{\left(I_{1295}+I_{1305}+I_{1270}\right)} \times 100 .
$$

A similar type of analysis can be performed for PP, in this case considering the characteristic vibrational bands located at 808 , related to PP's $\alpha$-monoclinic crystalline phase, and $840 \mathrm{~cm}^{-1}$, related to the amorphous part [12]:

$$
\begin{gathered}
I_{c P P}=\frac{I_{808}}{I_{808}+I_{840}} \times 100, \\
I_{a P P}=\frac{I_{840}}{I_{808}+I_{840}} \times 100 .
\end{gathered}
$$


The numeric results of both PE and PP's amorphous and crystalline content analysis are compiled in Table 1. Typical Raman spectra are presented respectively for the PE and PP-based foams in Figures 6 and 7.
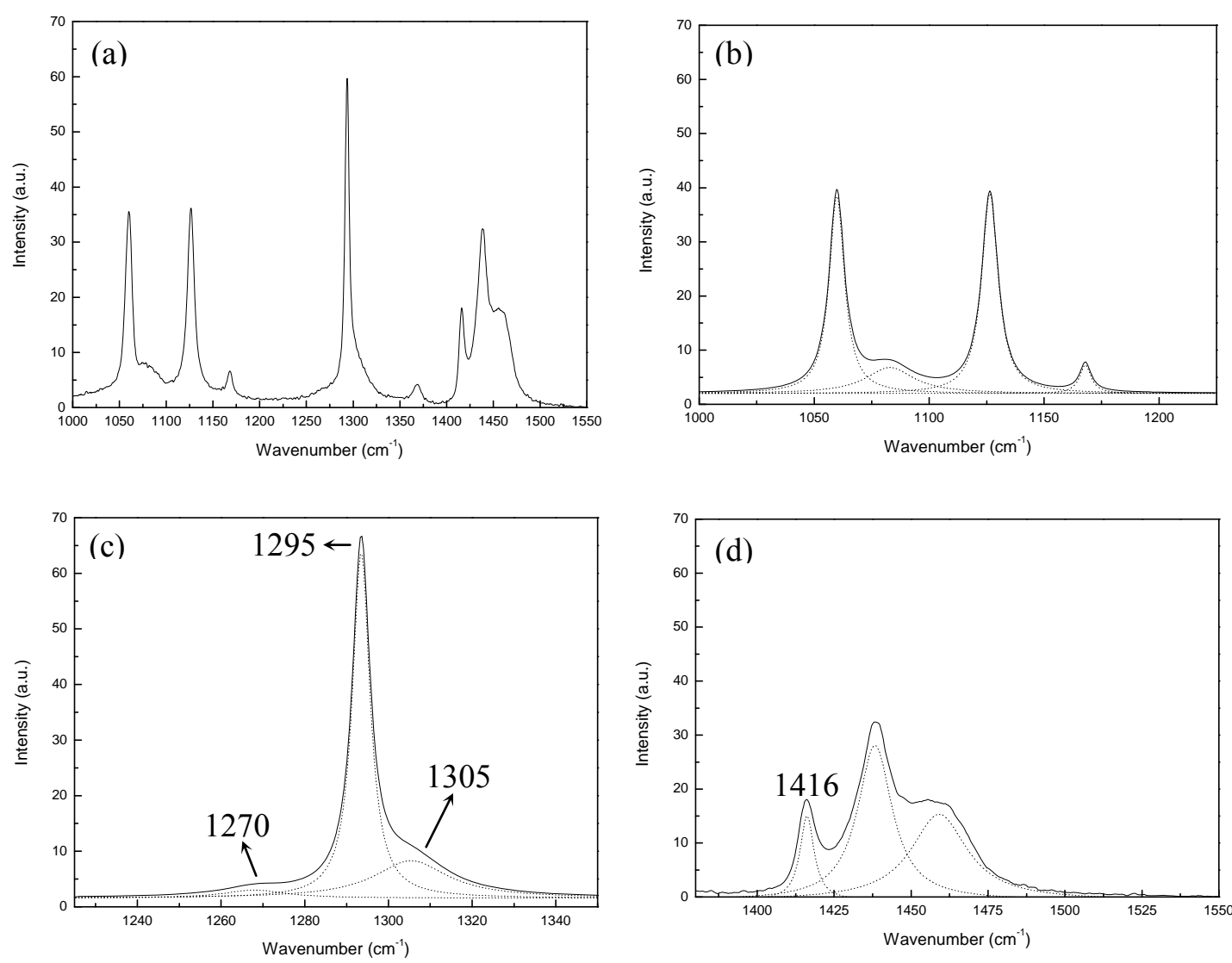

Figure 6. (a) Typical Raman spectrum of the PE-based foam and (b), (c) and (d) details showing Lorentzian and Gaussian curves convolution analysis.
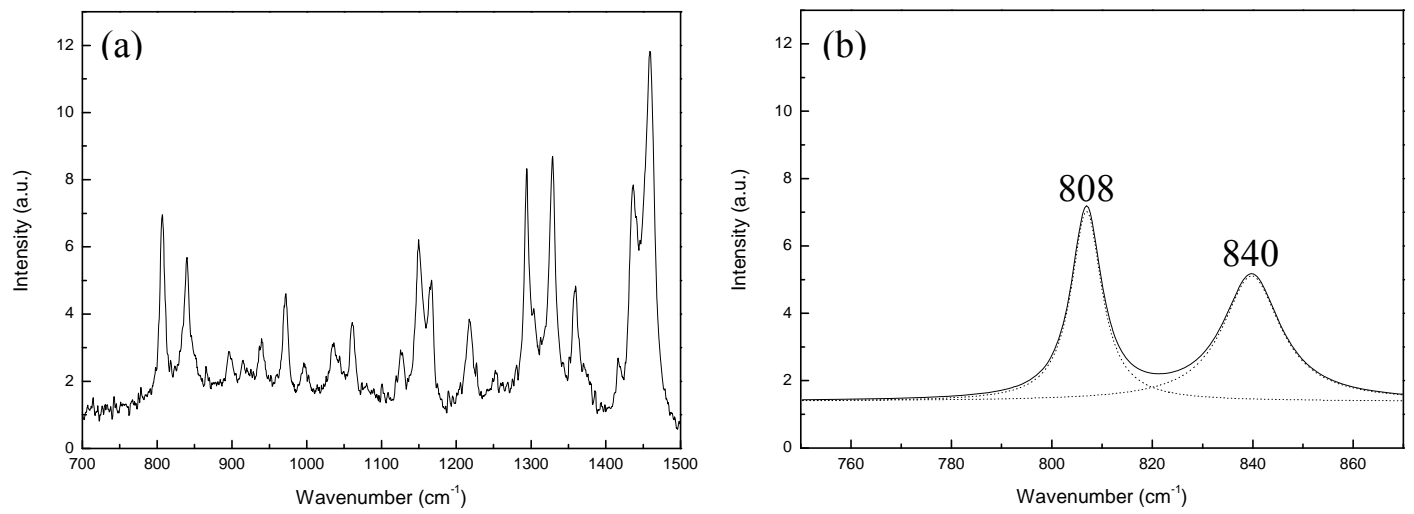

Figure 7. (a) Typical Raman spectrum of the PP-based foam and (b) Lorentzian and Gaussian curves convolution analysis of the 808 and $840 \mathrm{~cm}^{-1}$ peaks. 
Table 1. Crystalline and amorphous fractions of the PE and PP-based foams obtained by Raman

\begin{tabular}{|c|c|c|c|c|c|c|}
\hline $\begin{array}{l}\text { Material } \\
\text { code }\end{array}$ & Plane & Direction & $I_{c}(\%)$ & $I_{a}(\%)$ & \multicolumn{2}{|c|}{$\mathbf{I}_{1416} / \mathbf{I}_{1305}\left(\mathbf{I}_{1416} / \mathbf{I}_{1305}\right)_{\text {norm }}$} \\
\hline \multirow{3}{*}{ PE } & $(\mathrm{MD}, \mathrm{TD})$ & ED & 45.2 & 11.1 & 1.6 & 0.7 \\
\hline & (MD,ED) & TD & 47.4 & 10.1 & 2.4 & 1.0 \\
\hline & $(\mathrm{TD}, \mathrm{ED})$ & MD & 36.0 & 9.7 & 2.2 & 0.9 \\
\hline \multicolumn{7}{|c|}{$\mathbf{I}_{808} / \mathbf{I}_{840} \quad\left(\mathbf{I}_{808} / \mathbf{I}_{840}\right)_{\text {norm }}$} \\
\hline \multirow{3}{*}{ PP } & $(\mathrm{MD}, \mathrm{TD})$ & ED & 58.4 & 41.6 & 1.5 & 1.0 \\
\hline & $(\mathrm{MD}, \mathrm{ED})$ & TD & 49.7 & 50.3 & 1.4 & 0.9 \\
\hline & (TD,ED) & MD & 61.0 & 39.0 & 1.4 & 0.9 \\
\hline
\end{tabular}

In PE foams, the values of the amorphous fraction are quite similar for all the directions, whereas the lowest value of the crystalline fraction is found in MD direction ((TD,ED) plane). Concerning the PP-based foam, the amorphous fraction is located preferentially in TD ((MD,ED) plane) and thus the crystalline fraction is more present in the two other directions (ED and MD).

In order to assess possible preferential crystalline orientations due to processing, the relation between the crystalline and amorphous characteristic peaks was determined for the PE and PP foams (two last columns of Table 1). In the case of the PE foams, a preferential TD crystalline orientation was observed, considerable differences being only assessed regarding the ED direction, the foamed material showing a lower value of the normalized crystalline intensity $\left(\left(I_{1416} / I_{1305}\right)_{\text {norm }}\right)$.

No considerable differences were observed for the PP-based foams, the material showing almost the same value of $\left(I_{808} / I_{840}\right)_{\text {norm }}$ for all considered directions.

4.2.2. Local crystalline orientation by micro-Raman. The same type of numeric analysis was performed on both PE and PP-based foams with a spatial resolution of $1 \mu \mathrm{m}^{2}$ by pointing the Raman beam at two different cell zones: edge centre and cell face (Figure 1(b)). The results are compiled in Table 2.

Table 2. Crystalline and amorphous fractions of the PE and PP-based foams obtained by microRaman

\begin{tabular}{|c|c|c|c|c|c|c|c|}
\hline $\begin{array}{c}\text { Material } \\
\text { code }\end{array}$ & $\begin{array}{c}\text { Measured } \\
\text { zone }\end{array}$ & Plane & Direction & $\mathbf{I}_{\mathrm{c}}(\%)$ & $I_{a}(\%)$ & $\mathbf{I}_{\mathbf{1 4 1 6}} / \mathbf{I}_{\mathbf{1 3 0 5}}$ & $\left(\mathbf{I}_{1416} / \mathbf{I}_{1305}\right)_{\text {norm }}$ \\
\hline \multirow{6}{*}{ PE } & \multirow{3}{*}{ Edge centre } & (MD,TD) & ED & 48.2 & 9.3 & 2.3 & 0.9 \\
\hline & & (MD,ED) & TD & 53.2 & 9.5 & 2.5 & 1.0 \\
\hline & & (TD,ED) & MD & 37.9 & 9.6 & 1.8 & 0.7 \\
\hline & \multirow{3}{*}{ Cell face } & (MD,TD) & ED & 45.7 & 10.9 & 1.9 & 1.0 \\
\hline & & (MD,ED) & TD & 47.4 & 10.1 & 2.1 & 1.0 \\
\hline & & $(\mathrm{TD}, \mathrm{ED})$ & MD & 34.1 & 9.9 & 1.5 & 0.8 \\
\hline & & & & & & $\mathbf{I}_{\mathbf{8 0 8}} / \mathbf{I}_{\mathbf{8 4 0}}$ & $\left(\mathbf{I}_{808} / \mathbf{I}_{840}\right)_{\text {norm }}$ \\
\hline \multirow{6}{*}{ PP } & \multirow{3}{*}{ Edge centre } & $(\mathrm{MD}, \mathrm{TD})$ & ED & 51.9 & 48.1 & 1.1 & 0.7 \\
\hline & & (MD,ED) & TD & 60.9 & 39.1 & 1.6 & 1.0 \\
\hline & & (TD,ED) & MD & 58.9 & 41.1 & 1.4 & 0.9 \\
\hline & \multirow{3}{*}{ Cell face } & $(\mathrm{MD}, \mathrm{TD})$ & ED & 60.3 & 39.7 & 1.5 & 1.0 \\
\hline & & (MD,ED) & TD & 59.5 & 40.5 & 0.7 & 0.4 \\
\hline & & $(\mathrm{TD}, \mathrm{ED})$ & MD & 61.2 & 38.8 & 1.6 & 1.0 \\
\hline
\end{tabular}


Concerning PE results, we observe a higher crystalline fraction of the chains in the flow direction, leading to a more marked orientation in the edge zone (more sensible to material drawing) than in the cell faces (whose orientation is mainly expansion-dependent). These results differ from those found in foams obtained by a physical foaming process using an autoclave, which showed an opposite trend due to their highly isometric-like cellular structure [7]. The particularities of our foaming process, with combined orientation effects of both extrusion drawing and foam expansion is the reason behind this apparently incoherent opposite behaviour. A higher crystalline fraction is observed for both the edge centre and cell face in the plane (MD,ED), that is, in TD direction.

For PP, measurements done in the edge centre show a slightly higher crystalline fraction of the polymeric chains in the plane (MD,ED) than in (TD,ED) and considerably higher than in the plane $(M D, T D)$. Measurements performed on the cell face gives almost identical values for the three analyzed directions.

\subsection{Mechanical anisotropy}

The visualization of both MD and TD tensile curves of the polyethylene foam (Figure 8) shows a marked elastic behaviour throughout the trial, failure coming at moderate plastic deformation values when compared to those of the PP foams. The tensile strength values were higher in MD direction (yield stresses of $0.22 \mathrm{MPa}$ for PE and 1.01 MPa for PP) than in TD (PE: 0.06 MPa; PP: $0.59 \mathrm{MPa}$ ). Due to their intrinsic nature, polypropylene foams show an extensive plastic deformation tensile behaviour and tensile strength values higher than that of the polyethylene ones.

Although showing almost the same crystalline orientation in MD than TD if we consider the average values obtained using the $\times 10$ microscope objective (Table 1 ), considerable differences were found regarding PP's relative crystalline orientation in the cell faces, MD giving a considerably higher (almost two times higher) crystalline orientation than TD. While edges are commonly used to assess relative crystalline orientations induced due to processing (extrusion flow), cell faces are related to orientations induced by the combined effects of material stretching due to processing and biaxial stretching due to the foam's expansion at the exit of the die. So, it seems that the combination of a highly MD-stretched material (Figure 4(d)) and considerable foaming of the PP-based matrix results in an MD crystalline-oriented PP matrix, ultimately resulting in the higher tensile properties observed for the MD samples regarding the TD ones (Figure 8).

The particular elastic tensile behaviour of the PE-based foams restricted possible differences between MD and TD directions. So, although in all cases presenting slightly higher relative crystallinities in TD than in MD (Table 2), the MD specimens showed a higher tensile strength, probably due to predominant cell stretching under these loading conditions. 


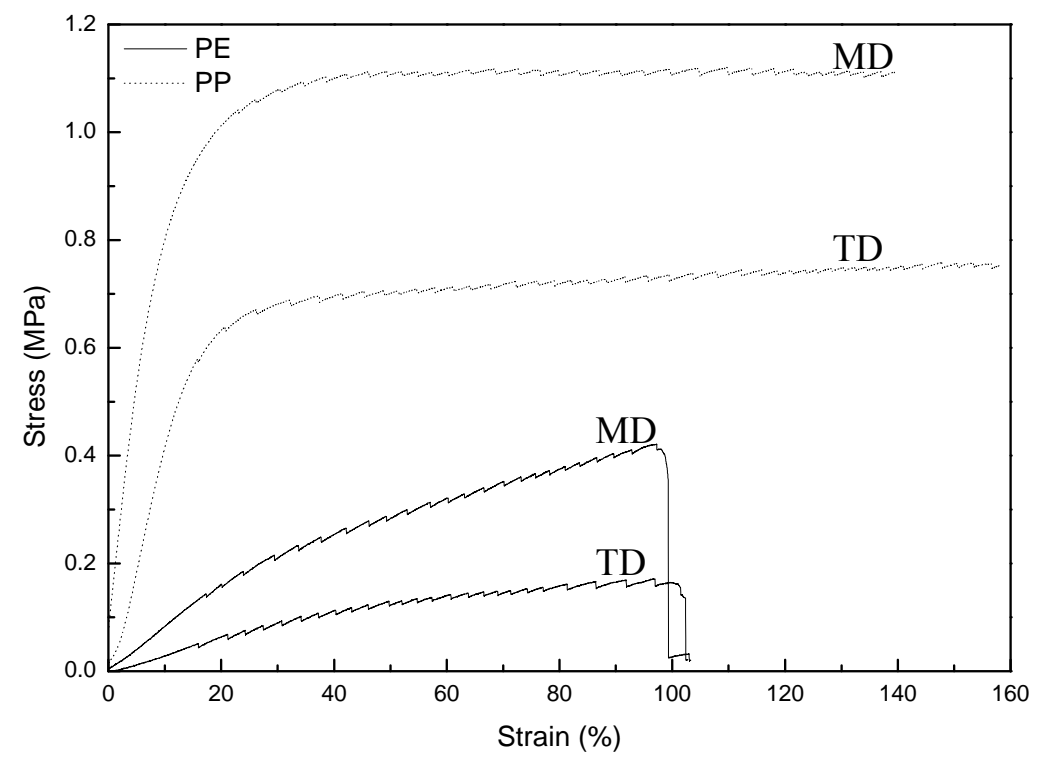

Figure 8. Comparative between the typical tensile curves of the PE and PP-based foams in MD and TD directions.

\section{Conclusions}

The inner microstructure of two polyolefin-based foams was studied using Raman spectroscopy and related to the direction-dependent tensile behaviour of the materials.

Previously, DSC and FT-IR analyses were carried out, revealing that the PE-based foam contained a small amount of an ethylene vinyl acetate copolymer (EVA) and the PP-based one an ethylenic copolymer with a low melting point.

Micro-Raman was used to assess possible crystalline and amorphous content induced orientations due to processing and foaming expansion. A relative crystalline orientation parameter was proposed for both foams, in both cases considering the most characteristic respective Raman crystalline peak (PE: $1416 \mathrm{~cm}^{-1}$ and PP: $808 \mathrm{~cm}^{-1}$ ). It was found that PE foams showed slightly higher TD relative crystalline orientations in both the edges as well as cell faces, while the PP foams showed a marked MD crystalline orientation, mainly observed in the cell faces, and attained to the combined effects of polymer and biaxial stretching during processing and foam growth.

Alongside the expected higher stiffness, tensile strength and ductility of the PP-based foams regarding PE, tensile tests showed how the induced MD crystalline orientation of the PP foams resulted in clearly higher tensile strengths than in TD.

Although further experiments are clearly needed to further elucidate possible foam microstructure influences in the mechanical behaviour of these foamed materials, for instance considering different expansion ratios, in order to analyze MD and TD differences due to the polymer's biaxial stretching during expansion, these preliminary results clearly showed how the knowledge of the inner microstructure is crucial to explain the direction-dependent mechanical properties of these materials.

\section{Acknowledgments}

Financial assistance from the Spanish Ministry of Science and Education for the project MAT200762956 is gratefully acknowledged. 


\section{References}

[1] Gibson L J and Ashby M F 1988 Cellular solids: structure and properties (Oxford: Pergamon Press)

[2] Hilyard N C and Cunningham A 1994 Low density cellular plastics: physical basis of behaviour (London: Chapman \& Hall)

[3] Klempner D and Firsch C 1991 Polymeric foams (Munich: Hanser Publishers)

[4] Velasco J I, Morhain C, Martínez A B, Rodríguez-Pérez M A and de Saja J A 2002 Polymer 43 6805

[5] Zipper P and Djoumaliisky S 2002 Macromol. Symp. 181421

[6] Almanza O, Rodríguez-Pérez M A, Chernev B, de Saja J A and Zipper P 2005 Eur. Polym. J. 41599

[7] Rodríguez-Pérez M A, Campo-Arnáiz R A, Aroca R F and de Saja J A 2005 Polymer 4612093

[8] Almanza O, Rodríguez-Pérez M A and de Saja J A 2001 Polymer 432671

[9] Socrates G 2004 Infrared and Raman Characteristic Group Frequencies, Tables and Charts (John Wiley and Sons, Ltd)

[10] Ströbl C R and Hagedorn W 1978 J. Polym. Sci. Polym. Phys. 161181

[11] Mandelkern L, Alamo R G and Kennedy M A 1990 Macromolecules 234721

[12] Nielsen A S, Batchelder D N and Pyrz R 2002 Polymer 432671 